This is an Accepted Manuscript of an article published by Taylor \& Francis in "Linear and multilinear algebra" on $08^{\text {th }}$ July 2016 , available online:

http://www.tandfonline.com/doi/full/10.1080/03081087.2016.1256945 


\title{
Effective resistances and Kirchhoff Index in subdivision networks
}

\author{
Ángeles Carmona, Margarida Mitjana, Enric Monsó \\ Departament de Matemàtiques \\ Universitat Politècnica de Catalunya. Spain
}

\begin{abstract}
We define a subdivision network $\Gamma^{S}$ of a given network $\Gamma$, by inserting a new vertex in every edge, so that each edge is replaced by two new edges with conductances that fulfill electrical conditions on the new network. In this work, we firstly obtain an expression for the Green kernel of the subdivision network in terms of the Green kernel of the base network. Moreover, we also obtain the effective resistance and the Kirchhoff index of the subdivision network in terms of the corresponding parameters on the base network. Finally, as an example, we carry out the computations in the case of a wheel.
\end{abstract}

Keywords: Resistance distance, Green kernel, Kirchhoff Index, Subdivision network MSC: 31C20, 15A09, 34B45

\section{Introduction}

Many recent papers are devoted to the study of different parameters of the subdivision graphs. For instance, Chen in [9], obtained a formula for the effective resistances of the subdivision graph in terms of the effective resistances of the original graph by using some nice sum rules. The Kirchhoff index of the subdivision graph is considered in different works under several hypothesis such as regular graphs in [12], general graphs in [16], or operations between graphs that involve the subdivision concept as well, see $[6,14]$ for instance.

In [18], the author extended the results in [6] and computed the Kirchhoff index of subdivision graph in terms of the Kirchhoff index, the multiplicative degree-Kirchhoff index, the additive degreeKirchhoff index, the number of vertices, and the number of edges of $\Gamma$. Simultaneously, Sun et alt. gave the formulae for the Kirchhoff index in terms of a $\{1\}$-inverse of the combinatorial Laplacian, see [16]. The case of some composite graphs is treated in [14], in particular the edge-corona and deletion of former edges graph (which is a way to achieve the subdivision graph).

$\operatorname{In}[3,4]$ the authors introduced a generalization of the Kirchhoff index of a finite network that consists in defining the generalitzation of the effective resistance between any pair of vertices with respect to a value $\lambda \geq 0$ and a vertex weight $\omega$ on the vertex set and this concept has been used to the study of composite networks, $[1,5]$.

The Kirchhoff Index is defined as the sum of all effective resistances between any pair of vertices of a network and it is also known as the total effective resistance, see [13]. It measures how well connected a network is. It was introduced in Chemistry as a better alternative to other 
parameters used for discriminating among different molecules with similar shapes and structures, see [15]. It also arises in other fields as, for instance electrical networks where is in connection with power dissipation and in a Markov chain scenario, where it describes the average hitting time, see [13] and references therein.

The most important property of the effective resistance between vertices, [15], is that it defines a distance function between vertices in a graph. It is a valuable tool in the analysis of network problems. The resistance distance has been extensively studied not only in mathematical papers but also in physical and chemical, see [19].

In the present paper, we introduce the subdivision of a network. Our approach consists in interpreting a network as an electric circuit, and hence each edge has got assigned a positive number that corresponds with the conductance of a wire connecting two nodes, its inverse is the resistance. When we perform the subdivision operation we interpret that we introduce a rheostat in every edge, that is a device that may change the resistance without opening the circuit in which it is connected. Thus, we decompose each edge in two new edges taking into account electrical compatibility of the circuit, specifically, the series sum rule for resistances. As a consequence, we would get that after the subdivision process, the effective resistance between any pair of old vertices should remain unchanged.

Our methodology comes from discrete Potential theory and hence, we express all the parameters in terms of the Green kernel of the network. That can be seen in matrix terms, as the computation of the Group inverse of the combinatorial Laplacian of a subdivision network in terms of the Group inverse of the combinatorial Laplacian of the base network.

In Section 2 we first obtain a solution of the Poisson problem in the subdivision network in terms of the solution of an appropriate Poisson problem on the base network and hence we compute the Green kernel of the subdivision network. Next, we give an expression for the effective resistance between any pair of vertices of the subdivision network and its corresponding Kirchhoff index. For all the results we compare ours with the previously known for the case of graphs, and in particular for $k$-regular graphs.

The last section contains the expressions for the Green kernel, the effective resistance and the Kirchhoff index of the subdivision network of a wheel as an illustration of the obtained results.

We end the present section by introducing the basic notation and results.

In the whole work, a network is the triplet $\Gamma=(V, E, c)$ where $(V, E)$ stands for a finite and connected graph, without loops nor multiple edges; and $c: V \times V \longrightarrow[0,+\infty)$ is a symmetric function called conductance satisfying $c(x, y)>0$ iff $x \sim y$ which means that $\{x, y\} \in E$. Let $n$ be the number of nodes and $m$ the number of edges.

On the other hand, $\mathcal{C}(V)$ is the set of real functions on $V$. For any vertex $x \in V, \varepsilon_{x} \in \mathcal{C}(V)$ is the Dirac function at $x$ and $k \in \mathcal{C}(V)$ defined as $k(x)=\sum_{y \in V} c(x, y)$, is the degree of $x$. The standard inner product in $\mathcal{C}(V)$ is denoted by $\langle\cdot, \cdot\rangle$; that is, if $u, v \in \mathcal{C}(V)$ then, $\langle u, v\rangle=\sum_{x \in V} u(x) v(x)$.

The Laplacian of $\Gamma$ is the linear operator $\mathcal{L}: \mathcal{C}(V) \longrightarrow \mathcal{C}(V)$ defined, for each $u \in \mathcal{C}(V)$ and $x \in V$ as

$$
\mathcal{L}(u)(x)=\sum_{y \in V} c(x, y)(u(x)-u(y)) .
$$

The Laplacian of $\Gamma$ is a self-adjoint and positive semi-definite operator. Moreover, $\mathcal{L}(u)=0$ iff $u$ is 
constant and hence, $\operatorname{ker}(\mathcal{L})=\operatorname{span}(1)$, where $1 \in \mathcal{C}(V)$ is the function that assigns 1 to any vertex. Therefore, $\mathcal{L}$ defines an isomorphism on $\operatorname{ker}(\mathcal{L})^{\perp}$.

A Poisson problem consists in, given $f \in \mathcal{C}(V)$, finding $u \in \mathcal{C}(V)$ such that

$$
\mathcal{L}(u)=f \quad \text { on } \quad V .
$$

From the above properties, the Poisson equation has solution iff $\langle f, 1\rangle=0$, and there is a unique solution of (1) satisfying $\langle u, 1\rangle=0$.

The operator that assigns to every function $f \in \mathcal{C}(V)$ the unique solution of $\mathcal{L}(u)=f-\langle f, 1\rangle 1$, such that $\langle u, 1\rangle=0$, is called the Green operator and it is denoted by $\mathcal{G}$. The operator $\mathcal{G}$ is selfadjoint and positive semi-definite. Then the symmetric function $G$, defined as $G(x, y)=\mathcal{G}\left(\varepsilon_{y}\right)(x)$ for every pair $x, y \in V$, is called the Green kernel on $V$. Moreover, $\mathcal{G}(f)(x)=\sum_{x, y \in V} G(x, y) f(y)$ and $\langle\mathcal{G}(f), f\rangle=0$ iff $f=a 1, a \in \mathbb{R}$.

The relation between an integral operator and its associated kernel enables us to characterize the Green kernel for $\Gamma$ as solutions of suitable boundary value problems. For all $y \in V$, the function $G_{y}=G(\cdot, y)$ is characterized by equations

$$
\mathcal{L}\left(G_{y}\right)=\varepsilon_{y}-\frac{1}{n} 1 \quad \text { and } \quad\left\langle G_{y}, 1\right\rangle=0 .
$$

See [8] and references therein for more details. Notice that, if we label the vertices of $\Gamma$, both the Laplacian and the Green operator can be interpreted as matrices and hence, the Green kernel can be identified as the Group Inverse of the combinatorial Laplacian.

The effective resistance between vertices $x$ and $y$ is defined as $R(x, y)=u(x)-u(y)$, where $u \in \mathcal{C}(V)$ is any solution of the Poisson problem $\mathcal{L}(u)=\varepsilon_{x}-\varepsilon_{y}$. The effective resistance can be interpreted as the voltage measured in the nodes $x$ and $y$ when a unitary current is applied between them. Actually, $R$ defines a distance on $\Gamma$, usually referred as resistive distance, see $[2,15]$, and gives a measure of how much two different nodes of a network are connected. Thus, the more well connected are vertices $x$ and $y$, the less is $R(x, y)$. Moreover, for any $x, y \in V$ the following relation holds

$$
R(x, y)=G(x, x)+G(y, y)-2 G(x, y) .
$$

The Kirchhoff Index $\mathrm{k}$ of a network $\Gamma$, also called its total resistance, is defined as

$$
\mathrm{k}=\frac{1}{2} \sum_{x, y \in V} R(x, y)=n \sum_{x \in V} G(x, x)
$$

and gives a measure of the global connectivity of the network. The Kirchhoff index is a descriptor of the structure of the network and exhibits many interesting interpretations, see $[13,17]$.

\section{The Poisson Problem on a Subdivision Network}

A subdivision network $\Gamma^{S}=\left(V^{S}, E^{S}, c^{S}\right)$ of a given network $\Gamma=(V, E, c)$, is obtained by inserting a new vertex in every edge, so that each edge $\{x, y\} \in E$ is replaced by two new edges, say $\left\{x, v_{x y}\right\}$ and $\left\{y, v_{x y}\right\}$ where $v_{x y}$ is the new inserted vertex. We denote by $V^{\prime}$ the new vertex set assuming 
that, $v_{x y}=v_{y x}$. Thus, $V^{S}=V \cup V^{\prime}$, the order of the subdivision network is $n+m$, whereas the size is $2 m$. Moreover, according to the well-known rule that express the equivalent resistance of two resistors connected in series, we define the conductance function $c^{S}: V^{S} \times V^{S} \longrightarrow[0,+\infty)$ by choosing, for every pair of adjacent vertices, non-null values $c^{S}\left(x, v_{x y}\right)$ and $c^{S}\left(y, v_{x y}\right)$ such that

$$
\frac{1}{c(x, y)}=\frac{1}{c^{S}\left(x, v_{x y}\right)}+\frac{1}{c^{S}\left(y, v_{x y}\right)} \text {. }
$$

The definition of $c^{S}$ cannot be misunderstood as all the edges in $E^{S}$ have both kind of vertices, one in $V$ and the other in $V^{\prime}$. Hence, by the sake of simplicity, it will be denoted as $c$. Moreover for each edge, there exist infinitely many different choices of conductances fulfilling (5), so that different choices will lead to different subdivision networks.

Up to our knowledge, the only case that has been studied in the literature, $([9,12,16,18])$, is $c(x, y)=c\left(x, v_{x y}\right)=c\left(y, v_{x y}\right)=1$, that not fulfills the electrical compatibility condition (5). In the present work, and in order to compare with the known results, we will consider as a particular case $c(x, y)=1$ and $c\left(x, v_{x y}\right)=c\left(y, v_{x y}\right)=2$ and we call it standard subdivision graph.

Observe that $\Gamma^{S}$ is also a connected, finite, with no loops, nor multiple edges network.

If $\mathcal{L}^{S}$ denotes the combinatorial Laplacian of $\Gamma^{S}$, then for any $u \in \mathcal{C}\left(V^{S}\right)$ we have that

$$
\begin{aligned}
\mathcal{L}^{S}(u)(x) & =\sum_{y \in V} c\left(x, v_{x y}\right)\left(u(x)-u\left(v_{x y}\right)\right), & & \text { for any } x \in V ; \\
\mathcal{L}^{S}(u)\left(v_{x y}\right) & =c\left(x, v_{x y}\right)\left(u\left(v_{x y}\right)-u(x)\right)+c\left(y, v_{x y}\right)\left(u\left(v_{x y}\right)-u(y)\right), & & \text { for any } v_{x y} \in V^{\prime} .
\end{aligned}
$$

The aim of this section is to obtain a solution of the Poisson problem in $\Gamma^{S}$ in terms of the solution of an appropriate Poisson problem on $\Gamma$.

It is helpful for the sequel to define, for each pair $x, y \in V$ with $x \sim y$, the coefficient

$$
\alpha(x, y)=\frac{c\left(x, v_{x y}\right)}{c\left(x, v_{x y}\right)+c\left(y, v_{x y}\right)}=\frac{c\left(x, v_{x y}\right)}{k\left(v_{x y}\right)}
$$

where $k\left(v_{x y}\right)=c\left(x, v_{x y}\right)+c\left(y, v_{x y}\right)$, is the degree of $v_{x y}$ in $\Gamma^{S}$. Notice that $\alpha(y, x)=1-\alpha(x, y)$. Moreover, $\alpha(x, y)$ is nothing else but the transition probability from $v_{x y}$ to $x$ of the reversible Markov chain associated with $\Gamma^{S}$. In additon, if $x \not y$ we define $\alpha(x, y)=\alpha(y, x)=0$. Notice that, for any $x, y$ is $\alpha(x, y)=\alpha(y, x)$ iff $c\left(x, v_{x y}\right)=c\left(y, v_{x y}\right)=2 c(x, y)$.

We also define, for each $h \in \mathcal{C}\left(V^{S}\right)$ and $u \in \mathcal{C}(V)$, the contraction of $h$ to $V, \underline{h} \in \mathcal{C}(V)$, as

$$
\underline{h}(x)=h(x)+\sum_{y \sim x} \alpha(x, y) h\left(v_{x y}\right), \quad x \in V,
$$

and, the extension of $u$ to $V^{S}$ with respect to $h, u^{h} \in \mathcal{C}\left(V^{S}\right)$, as

$$
\begin{aligned}
u^{h}(x) & =u(x), & & \text { for all } x \in V ; \\
u^{h}\left(v_{x y}\right) & =\frac{h\left(v_{x y}\right)}{k\left(v_{x y}\right)}+\alpha(x, y) u(x)+\alpha(y, x) u(y), & & \text { for all } v_{x y} \in V^{\prime} .
\end{aligned}
$$

Notice that the extension of $u$ to $V^{S}$ with respect to $h$, has been defined in order to satisfy $\mathcal{L}^{S}\left(u^{h}\right)\left(v_{x y}\right)=h\left(v_{x y}\right)$. 
Theorem 2.1. Given $h \in \mathcal{C}\left(V^{S}\right)$ such that $\left\langle h, 1_{V^{S}}\right\rangle=0$, then $\left\langle\underline{h}, 1_{V}\right\rangle=0$. Moreover, $\bar{u} \in \mathcal{C}\left(V^{S}\right)$ is a solution of the Poisson equation $\mathcal{L}^{S}(\bar{u})=h$ in $V^{S}$ iff $u=\bar{u}_{\mid V}$ is a solution of the Poisson equation $\mathcal{L}(u)=\underline{h}$ in $V$. In this case, the identity $\bar{u}=u^{h}$ holds.

Proof. Firstly we note that $\left\langle\underline{h}, 1_{V}\right\rangle=\left\langle h, 1_{V^{S}}\right\rangle$ as

$$
\sum_{x \in V} \underline{h}(x)=\sum_{x \in V} h(x)+\sum_{x \in V} \sum_{y \sim x} \alpha(x, y) h\left(v_{x y}\right)=\sum_{x \in V} h(x)+\sum_{v_{x y} \in V^{\prime}} h\left(v_{x y}\right) .
$$

So the first statement holds.

Given $h \in \mathcal{C}\left(V^{S}\right)$ such that $\left\langle h, 1_{V^{S}}\right\rangle=0$ and $\bar{u}$ a solution of the Poisson equation $\mathcal{L}^{S}(\bar{u})=h$ in $V^{S}$, then

$$
\begin{aligned}
h\left(v_{x y}\right) & =c\left(x, v_{x y}\right)\left(\bar{u}\left(v_{x y}\right)-\bar{u}(x)\right)+c\left(y, v_{x y}\right)\left(\bar{u}\left(v_{x y}\right)-\bar{u}(y)\right), & & \text { for any } v_{x y} \in V^{\prime} ; \\
h(x) & =\sum_{y \sim x} c\left(x, v_{x y}\right)\left(\bar{u}(x)-\bar{u}\left(v_{x y}\right)\right), & & \text { for any } x \in V .
\end{aligned}
$$

The first identity implies $\bar{u}\left(v_{x y}\right)=u^{h}\left(v_{x y}\right)$, assuming $u=\bar{u}_{\mid V}$. Then, substituting the expression of $\bar{u}\left(v_{x y}\right)$ in the second one, we obtain that

$$
\begin{aligned}
\mathcal{L}^{S}(\bar{u})(x) & =\sum_{y \sim x} c\left(x, v_{x y}\right)\left(\bar{u}(x)-\frac{h\left(v_{x y}\right)}{k\left(v_{x y}\right)}-\alpha(x, y) \bar{u}(x)-\alpha(y, x) \bar{u}(y)\right) \\
& =\sum_{y \sim x} c\left(x, v_{x y}\right) \alpha(y, x)(\bar{u}(x)-\bar{u}(y))-\sum_{y \sim x} \frac{c\left(x, v_{x y}\right)}{k\left(v_{x y}\right)} h\left(v_{x y}\right) \\
& =\sum_{y \sim x} c(x, y)(u(x)-u(y))-\sum_{y \sim x} \alpha(x, y) h\left(v_{x y}\right) \\
& =\mathcal{L}(u)(x)-\underline{h}(x)+h(x),
\end{aligned}
$$

for every $x \in V$.

Therefore, $\mathcal{L}^{S}(\bar{u})=h$ in $V^{S}$ iff $\mathcal{L}(u)=\underline{h}$ in $V$.

Next result shows how to obtain the unique solution of a Poisson problem on the subdivision network $\Gamma^{S}$ orthogonal to $1_{V^{S}}$.

Corollary 2.2. Given $h \in \mathcal{C}\left(V^{S}\right)$, such that $\left\langle h, 1_{V^{S}}\right\rangle=0$, let $\underline{h} \in \mathcal{C}(V)$ be its contraction to $V$, $u \in \mathcal{C}(V)$ be the unique solution of $\mathcal{L}(u)=\underline{h}$ that satisfies $\left\langle u, 1_{V}\right\rangle=0$ and the constant

$$
\lambda=-\frac{1}{(n+m)} \sum_{x \sim y} \frac{h\left(v_{x y}\right)}{k\left(v_{x y}\right)}-\frac{1}{(n+m)} \sum_{x \sim y}[\alpha(x, y) u(x)+\alpha(y, x) u(y)] .
$$

Then, $u^{\perp}=u^{h}+\lambda$ is the unique solution of $\mathcal{L}^{S}\left(u^{\perp}\right)=h$ that satisfies $\left\langle u^{\perp}, 1_{V^{S}}\right\rangle=0$.

Proof. As two solutions differ on a constant, we have that $u^{\perp}=u^{h}+\gamma 1_{V^{S}}, \gamma \in \mathbb{R}$. Then,

$$
\begin{aligned}
0 & =\left\langle u^{\perp}, 1_{V^{S}}\right\rangle=\left\langle u^{h}, 1_{V^{S}}\right\rangle+(n+m) \gamma=\sum_{x \in V} u(x)+\sum_{x \sim y} u^{h}\left(v_{x y}\right)+(n+m) \gamma \\
& =\sum_{x \sim y} \frac{h\left(v_{x y}\right)}{k\left(v_{x y}\right)}+\sum_{x \sim y}(\alpha(x, y) u(x)+\alpha(y, x) u(y))+(n+m) \gamma,
\end{aligned}
$$

because $\left\langle u, 1_{V}\right\rangle=0$, and the result follows taking $\gamma=\lambda$. 


\section{The Green kernel of a subdivision network}

Taking into account the relation between Poisson problems on $\Gamma^{S}$ and $\Gamma$, we obtain the expression of the Green kernel of a subdivision network, $G^{S}$, in terms of Green's kernel of the base network. From now on we consider the function on $\mathcal{C}(V), \pi^{S}(x)=\sum_{y \sim x} \alpha(x, y)$ and the constant

$$
\beta=\frac{1}{(n+m)^{2}} \sum_{x, y \in V} G(x, y) \pi^{S}(x) \pi^{S}(y)+\frac{1}{(n+m)^{2}} \sum_{x \sim y} \frac{1}{k\left(v_{x y}\right)} .
$$

Proposition 3.1. Let $\Gamma^{S}$ be the subdivison network of $\Gamma$, then for any $x, z \in V$ and $v_{x y}, v_{z t} \in V^{\prime}$, the Green kernel of $\Gamma^{S}$ is given by

$$
\begin{aligned}
G^{S}(x, z) & =G(x, z)-\frac{1}{n+m} \sum_{\ell \in V}[G(x, \ell)+G(z, \ell)] \pi^{S}(\ell)+\beta, \\
G^{S}\left(v_{x y}, z\right) & =\alpha(x, y) G(x, z)+\alpha(y, x) G(y, z) \\
& -\frac{1}{n+m} \sum_{\ell \in V}[\alpha(x, y) G(x, \ell)+\alpha(y, x) G(y, \ell)+G(z, \ell)] \pi^{S}(\ell)-\frac{1}{(n+m) k\left(v_{x y}\right)}+\beta, \\
G^{S}\left(v_{x y}, v_{z t}\right) & =\alpha(z, t)(\alpha(x, y) G(x, z)+\alpha(y, x) G(y, z))+\alpha(t, z)(\alpha(x, y) G(x, t)+\alpha(y, x) G(y, t)) \\
& -\frac{1}{n+m} \sum_{\ell \in V}[\alpha(x, y) G(x, \ell)+\alpha(y, x) G(y, \ell)+\alpha(z, t) G(z, \ell)+\alpha(t, z) G(t, \ell)] \pi^{S}(\ell) \\
& +\frac{\varepsilon_{v_{z t}}\left(v_{x y}\right)}{k\left(v_{x y}\right)}-\frac{1}{(n+m) k\left(v_{x y}\right)}-\frac{1}{(n+m) k\left(v_{z t}\right)}+\beta .
\end{aligned}
$$

Proof. Suppose $z \in V$, and let $h_{z}=\varepsilon_{z}-\frac{1}{n+m}$. Then, for every $x \in V$

$$
\underline{h}_{z}(x)=\varepsilon_{z}(x)-\frac{1}{n+m}-\frac{1}{n+m} \sum_{y \sim x} \alpha(x, y)=\varepsilon_{z}(x)-\frac{1}{n+m}\left(1+\pi^{S}(x)\right) .
$$

Hence, from Equation (2), the Poisson problem to solve is $\mathcal{L}\left(u_{z}\right)=\underline{h}_{z}$, and, using the Green kernel for $\Gamma$, we obtain

$$
u_{z}(x)=G\left(\varepsilon_{z}\right)(x)-\frac{1}{n+m} \sum_{\ell \in V} G(x, \ell) \pi^{S}(\ell)=G(x, z)-\frac{1}{n+m} \sum_{\ell \in V} G(x, \ell) \pi^{S}(\ell) .
$$


Then, from Corollary 2.2

$$
\begin{aligned}
G_{z}^{S}(x) & =u_{z}^{h_{z}}(x)-\frac{1}{(n+m)} \sum_{r \sim s} \frac{h_{z}\left(v_{r s}\right)}{k\left(v_{r s}\right)}-\frac{1}{(n+m)} \sum_{r \sim s}\left[\alpha(r, s) u_{z}(r)+\alpha(s, r) u_{z}(s)\right] \\
& =G(x, z)-\frac{1}{n+m} \sum_{\ell \in V} G(x, \ell) \pi^{S}(\ell)+\frac{1}{(n+m)^{2}} \sum_{r \sim s} \frac{1}{k\left(v_{r s}\right)} \\
& -\frac{1}{(n+m)} \sum_{r \sim s} \alpha(r, s)\left[G(r, z)-\frac{1}{n+m} \sum_{\ell \in V} G(r, \ell) \pi^{S}(\ell)\right] \\
& -\frac{1}{(n+m)} \sum_{r \sim s} \alpha(s, r)\left[G(s, z)-\frac{1}{n+m} \sum_{\ell \in V} G(s, \ell) \pi^{S}(\ell)\right] \\
& =G(x, z)-\frac{1}{n+m} \sum_{\ell \in V}[G(x, \ell)+G(z, \ell)] \pi^{S}(\ell)+\frac{1}{(n+m)^{2}} \sum_{r, s} G(s, r) \pi^{S}(r) \pi^{S}(s) \\
& +\frac{1}{(n+m)^{2}} \sum_{r \sim s} \frac{1}{k\left(v_{r s}\right)} .
\end{aligned}
$$

Now, if $z \in V$ for every $v_{x y} \in V^{\prime}$

$$
\begin{aligned}
G_{z}^{S}\left(v_{x y}\right) & =\frac{h_{z}\left(v_{x y}\right)}{k\left(v_{x y}\right)}+\alpha(x, y) u_{z}(x)+\alpha(y, x) u_{z}(y) \\
& -\frac{1}{(n+m)} \sum_{r \sim s} \frac{h_{z}\left(v_{r s}\right)}{k\left(v_{r s}\right)}-\frac{1}{(n+m)} \sum_{r \sim s}\left[\alpha(r, s) u_{z}(r)+\alpha(s, r) u_{z}(s)\right] \\
& =-\frac{1}{(n+m) k\left(v_{x y}\right)}+\alpha(x, y) G(x, z)+\alpha(y, x) G(y, z) \\
& -\frac{1}{n+m} \sum_{\ell \in V}[\alpha(x, y) G(x, \ell)+\alpha(y, x) G(y, \ell)] \pi^{S}(\ell) \\
& +\frac{1}{(n+m)^{2}} \sum_{r \sim s} \frac{1}{k\left(v_{r s}\right)}-\frac{1}{(n+m)} \sum_{r \sim s} \alpha(r, s)\left[G(r, z)-\frac{1}{n+m} \sum_{\ell \in V} G(r, \ell) \pi^{S}(\ell)\right] \\
& -\frac{1}{(n+m)} \sum_{r \sim s} \alpha(s, r)\left[G(s, z)-\frac{1}{n+m} \sum_{\ell \in V} G(s, \ell) \pi^{S}(\ell)\right] \\
& =-\frac{1}{(n+m) k\left(v_{x y}\right)}+\alpha(x, y) G(x, z)+\alpha(y, x) G(y, z) \\
& -\frac{1}{n+m} \sum_{\ell \in V}[\alpha(x, y) G(x, \ell)+\alpha(y, x) G(y, \ell)+G(z, \ell)] \pi^{S}(\ell) \\
& +\frac{1}{(n+m)^{2}} \sum_{r \sim s} \frac{1}{k\left(v_{r s}\right)}+\frac{1}{(n+m)^{2}} \sum_{r, s} G(s, r) \pi^{S}(r) \pi^{S}(s) .
\end{aligned}
$$


Suppose now $v_{z t} \in V$, and let $h_{v_{z t}}=\varepsilon_{v_{z t}}-\frac{1}{n+m}$. Then, for every $x \in V$

$$
\begin{aligned}
\underline{h}_{v_{z t}}(x) & =\varepsilon_{v_{z t}}(x)-\frac{1}{n+m}+\sum_{y \in V} \alpha(x, y)\left(\varepsilon_{v_{z t}}\left(v_{x y}\right)-\frac{1}{n+m}\right) \\
& =-\frac{1}{n+m}\left(1+\pi^{S}(x)\right)+\alpha(z, t) \varepsilon_{z}(x)+\alpha(t, z) \varepsilon_{t}(x) .
\end{aligned}
$$

Hence, the Poisson problem to solve is $\mathcal{L}\left(u_{v_{z t}}\right)=\underline{h}_{v_{z t}}$, and, using Green's kernel for $\Gamma$, we obtain

$$
u_{v_{z t}}(x)=-\frac{1}{n+m} \sum_{\ell \in V} G(x, \ell) \pi^{S}(\ell)+\alpha(z, t) G(x, z)+\alpha(t, z) G(x, t) .
$$

Then, from Corollary 2.2

$$
\begin{aligned}
& G_{v_{z t}}^{S}\left(v_{x y}\right)=\frac{h_{v_{z t}}\left(v_{x y}\right)}{k\left(v_{x y}\right)}+\alpha(x, y) u_{v_{z t}}(x)+\alpha(y, x) u_{v_{z t}}(y) \\
& -\frac{1}{(n+m)} \sum_{r \sim s} \frac{h_{v_{z t}}\left(v_{r s}\right)}{k\left(v_{r s}\right)}-\frac{1}{(n+m)} \sum_{r \sim s}\left[\alpha(r, s) u_{v_{z t}}(r)+\alpha(s, r) u_{v_{z t}}(s)\right] \\
& =\frac{\varepsilon_{v_{z t}}\left(v_{x y}\right)}{k\left(v_{x y}\right)}-\frac{1}{(n+m) k\left(v_{x y}\right)} \\
& -\frac{1}{n+m} \sum_{\ell \in V}(\alpha(x, y) G(x, \ell)+\alpha(y, x) G(y, \ell)) \pi^{S}(\ell) \\
& +\alpha(z, t)(\alpha(x, y) G(x, z)+\alpha(y, x) G(y, z))+\alpha(t, z)(\alpha(x, y) G(x, t)+\alpha(y, x) G(y, t)) \\
& -\frac{1}{(n+m) k\left(v_{z t}\right)}+\frac{1}{(n+m)^{2}} \sum_{r \sim s} \frac{1}{k\left(v_{r s}\right)} \\
& -\frac{1}{n+m} \sum_{\ell \in V}(\alpha(z, t) G(z, \ell)+\alpha(t, z) G(t, \ell)) \pi^{S}(\ell) \\
& +\frac{1}{(n+m)^{2}} \sum_{r, s \in V} G(r, s) \pi^{S}(r) \pi^{S}(s) \\
& =\alpha(z, t)(\alpha(x, y) G(x, z)+\alpha(y, x) G(y, z))+\alpha(t, z)(\alpha(x, y) G(x, t)+\alpha(y, x) G(y, t)) \\
& -\frac{1}{n+m} \sum_{\ell \in V}(\alpha(x, y) G(x, \ell)+\alpha(y, x) G(y, \ell)+\alpha(z, t) G(z, \ell)+\alpha(t, z) G(t, \ell)) \pi^{S}(\ell) \\
& +\frac{\varepsilon_{v_{z t}}\left(v_{x y}\right)}{k\left(v_{x y}\right)}-\frac{1}{(n+m) k\left(v_{x y}\right)}-\frac{1}{(n+m) k\left(v_{z t}\right)}+\frac{1}{(n+m)^{2}} \sum_{r \sim s} \frac{1}{k\left(v_{r s}\right)} \\
& +\frac{1}{(n+m)^{2}} \sum_{r, s \in V} G(r, s) \pi^{S}(r) \pi^{S}(s) \text {. }
\end{aligned}
$$

In particular, if $\Gamma$ is a $k$-regular graph and we consider the standard subdivision graph; that is $c\left(x, v_{x y}\right)=c\left(y, v_{x y}\right)=2$, we get the following result. 
Corollary 3.2. Let $\Gamma^{S}$ be the standard subdivision graph of a $k$-regular graph, $\Gamma$; then for any $x, z \in V$ and $v_{x y}, v_{z t} \in V^{\prime}$, the Green kernel of $\Gamma^{S}$ is given by

$$
\begin{aligned}
G^{S}(x, z) & =G(x, z)+\frac{k}{2 n(2+k)^{2}} \\
G^{S}\left(v_{x y}, z\right) & =\frac{1}{2}(G(x, z)+G(y, z))-\frac{1}{n(2+k)^{2}}, \\
G^{S}\left(v_{x y}, v_{z t}\right) & =\frac{1}{4}\left(G(x, z)+G(y, z)+G(x, t)+G(y, t)+\varepsilon_{v_{z t}}\left(v_{x y}\right)\right)-\frac{(4+k)}{2 n(2+k)^{2}} .
\end{aligned}
$$

\section{Effective Resistances and Kirchhoff Index on subdivision net- works}

We are now concerned with the relation between effective resistances in a base network $\Gamma$ and the effective resistances, $R^{S}$, in a subdivision network $\Gamma^{S}$.

Theorem 4.1. Let $\Gamma=(V, E, c)$ be a network and $\Gamma^{S}=\left(V^{S}, E^{S}, c\right)$ its subdivision network, then

$$
\begin{aligned}
R^{S}(x, y) & =R(x, y), \\
R^{S}\left(x, v_{z t}\right) & =\frac{1}{k\left(v_{z t}\right)}+\alpha(z, t) R(x, z)+\alpha(t, z) R(x, t)-\alpha(z, t) \alpha(t, z) R(z, t), \\
R^{S}\left(v_{x y}, v_{z t}\right) & =\frac{1}{k\left(v_{x y}\right)}+\frac{1}{k\left(v_{z t}\right)} \\
& -\alpha(x, y) \alpha(y, x) R(x, y)-\alpha(z, t) \alpha(t, z) R(z, t) \\
& +\alpha(x, y) \alpha(z, t) R(x, z)+\alpha(x, y) \alpha(t, z) R(x, t) \\
& +\alpha(z, t) \alpha(y, x) R(y, z)+\alpha(y, x) \alpha(t, z) R(y, t), \text { for any } v_{x y} \neq v_{z t} .
\end{aligned}
$$

Proof. The proof is a direct consequence of Proposition 3.1 and Identity (3). Let us do the nontrivial case 2 . The case 3 , can be proved similarly. 


$$
\begin{aligned}
R^{S}\left(x, v_{z t}\right) & =G^{S}(x, x)+G^{S}\left(v_{z t}, v_{z t}\right)-2 G^{S}\left(x, v_{z t}\right) \\
& =G(x, x)-\frac{2}{n+m} \sum_{\ell \in V} G(x, \ell) \pi^{S}(\ell) \\
& +\alpha(z, t)(\alpha(z, t) G(z, z)+\alpha(t, z) G(t, z))+\alpha(t, z)(\alpha(z, t) G(z, t)+\alpha(t, z) G(t, t)) \\
& -\frac{2}{n+m} \sum_{\ell \in V}[\alpha(z, t) G(z, \ell)+\alpha(t, z) G(t, \ell)] \pi^{S}(\ell)+\frac{\varepsilon_{v_{z t}}\left(v_{z t}\right)}{k\left(v_{z t}\right)}-\frac{2}{(n+m) k\left(v_{z t}\right)} \\
& -2 \alpha(z, t) G(z, x)-2 \alpha(t, z) G(t, x) \\
& +\frac{2}{n+m} \sum_{\ell \in V}[\alpha(z, t) G(z, \ell)+\alpha(t, z) G(t, \ell)+G(x, \ell)] \pi^{S}(\ell)+\frac{2}{(n+m) k\left(v_{z t}\right)} \\
& =\frac{1}{k\left(v_{z t}\right)}+G(x, x)-2 \alpha(z, t) G(z, x)-2 \alpha(t, z) G(t, x) \\
& +\alpha(z, t)(\alpha(z, t) G(z, z)+\alpha(t, z) G(t, z))+\alpha(t, z)(\alpha(z, t) G(z, t)+\alpha(t, z) G(t, t)) \\
& =\frac{1}{k\left(v_{z t}\right)}+\alpha(z, t)[G(x, x)+G(z, z)-2 G(x, z)]+\alpha(t, z)[G(x, x)+G(t, t)-2 G(x, t)] \\
& -\alpha(t, z) \alpha(z, t)[G(z, z)+G(t, t)-2 G(z, t)],
\end{aligned}
$$

and hence, the result follows.

Observe that the effective resistance between vertices of the original network remains unchanged, as expected. In particular for the standard subdivision graph we get the following result, which coincides with the obtained in $[10,16,18]$, up to the factor 2 due to our (electrically compatible)-choice of the conductances.

Corollary 4.2. Let $\Gamma=(V, E, c)$ be a network and $\Gamma^{S}=\left(V^{S}, E^{S}, c\right)$ its standard subdivision network, then

$$
\begin{aligned}
R^{S}(x, y) & =R(x, y) \\
R^{S}\left(x, v_{z t}\right) & =\frac{1+2 R(x, z)+2 R(x, t)-R(z, t)}{4} \\
R^{S}\left(v_{x y}, v_{z t}\right) & =\frac{2-R(x, y)-R(z, t)+R(x, z)+R(x, t)+R(y, z)+R(y, t)}{4}, \text { for any } v_{x y} \neq v_{z t} .
\end{aligned}
$$

Next we obtain an expression for the Kirchhoff index of the subdivision network, $\mathrm{k}^{S}$, in terms of the Kirchhoff index, k, of the base network and other parameters.

Theorem 4.3. Let $\Gamma=(V, E, c)$ be a network and $\Gamma^{S}=\left(V^{S}, E^{S}, c\right)$ its subdivision network, then

$$
\begin{aligned}
\mathrm{k}^{S} & =\frac{n+m}{n} \mathrm{k}+(n+m) \sum_{x \in V} G(x, x) \pi^{S}(x)-\sum_{x, y \in V} G(x, y) \pi^{S}(x) \pi^{S}(y) \\
& -(n+m) \sum_{x \sim y} \alpha(x, y) \alpha(y, x) R(x, y)+(n+m-1) \sum_{x \sim y} \frac{1}{k\left(v_{x y}\right)} .
\end{aligned}
$$


Proof.

$$
\begin{aligned}
\mathrm{k}^{S} & =(n+m) \sum_{x \in V} G^{S}(x, x)+(n+m) \sum_{v_{x y} \in V^{\prime}} G^{S}\left(v_{x y}, v_{x y}\right) \\
& =\frac{(n+m)}{n} \mathrm{k}-2 \sum_{x \in V} \sum_{\ell \in V} G(x, \ell) \pi^{S}(\ell) \\
& +(n+m) \sum_{v_{x y} \in V^{\prime}}\left(\alpha(x, y)^{2} G(x, x)+2 \alpha(x, y) \alpha(y, x) G(y, x)+\alpha(y, x)^{2} G(y, y)\right) \\
& -2 \sum_{v_{x y} \in V^{\prime}} \sum_{\ell \in V}[\alpha(x, y) G(x, \ell)+\alpha(y, x) G(y, \ell)] \pi^{S}(\ell) \\
& +\sum_{x, y \in V} G(x, y) \pi^{S}(x) \pi^{S}(y)+(n+m-1) \sum_{x \sim y} \frac{1}{k\left(v_{x y}\right)} \\
& =\frac{n+m}{n} \mathrm{k}+(n+m) \sum_{x, y \in V}\left(\alpha(x, y)^{2} G(x, x)+\alpha(x, y) \alpha(y, x) G(y, x)\right) \\
& -\sum_{x, y \in V} G(x, y) \pi^{S}(x) \pi^{S}(y)+(n+m-1) \sum_{x \sim y} \frac{1}{k\left(v_{x y}\right)} \\
& =\frac{n+m}{n} \mathrm{k}+(n+m) \sum_{x \in V} G(x, x) \pi^{S}(x)-(n+m) \sum_{x \sim y} \alpha(x, y) \alpha(y, x) R(y, x) \\
& -\sum_{x, y \in V} G(x, y) \pi^{S}(x) \pi^{S}(y)+(n+m-1) \sum_{x \sim y} \frac{1}{k\left(v_{x y}\right)} .
\end{aligned}
$$

In particular, the Kirchhoff index of the standard subdivision graph has the following expression which, coincides with [16, Th 3.1]. In the case of $k$-regular graph the result coincides with [12, Th 3.5].

Corollary 4.4. Let $\Gamma^{S}$ be the standard subdivision network of a graph, $\Gamma$; then

$$
\mathrm{k}^{S}=\frac{n+m}{n} \mathrm{k}+(n+m) \sum_{x \in V} G(x, x) \pi^{S}(x)-\sum_{x, y \in V} G(x, y) \pi^{S}(x) \pi^{S}(y)+\frac{m^{2}-n^{2}+n}{4} .
$$

In particular, if $\Gamma$ is $k$-regular

$$
\mathrm{k}^{S}=\frac{(k+2)^{2}}{4} \mathrm{k}+\frac{\left(k^{2}-4\right) n^{2}+4 n}{16} .
$$

\section{Subdivision network of a wheel}

In order to illustrate the above results, we consider the wheel network with constant conductances and a subdivision of it. Let $W_{n}$ be the wheel network with vertex set $V=\left\{x_{0}, x_{1}, \ldots, x_{n}\right\}$, where $x_{0}$ has degree $n$, and conductances $c\left(x_{0}, x_{i}\right)=a>0$ for any $i=1, \ldots, n, c=c\left(x_{i}, x_{i+1}\right)$ if $i=1, \ldots, n-1$ and $c=c\left(x_{n}, x_{1}\right)$, as can be seen in Figure 1. For the sake of simplicity we consider that $x_{n+1}=x_{1}$. 


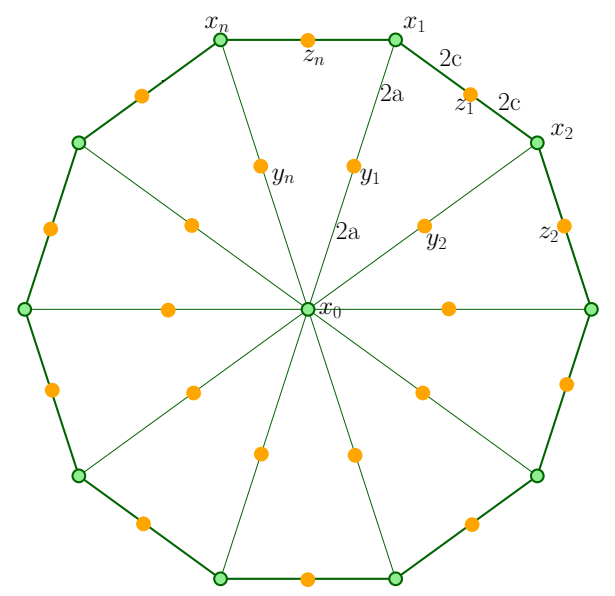

Figure 1: Subdivision network of a wheel of $n+1$ vertices

It is known, see for instance [8], that the Green function of $W_{n}$ is

$$
\begin{aligned}
G\left(x_{0}, x_{0}\right) & =\frac{n}{a(n+1)^{2}}, \\
G\left(x_{0}, x_{i}\right) & =\frac{-1}{a(n+1)^{2}}, \quad i=1, \ldots, n, \\
G\left(x_{i}, x_{j}\right) & =-\frac{n+2}{a(n+1)^{2}}+\frac{U_{n-1-|i-j|}(p)+U_{|i-j|-1}(p)}{2 c\left(T_{n}(p)-1\right)}, \quad i, j=1, \ldots, n,
\end{aligned}
$$

where $p=1+\frac{a}{2 c}$ and $U_{\ell}(x), T_{\ell}(x)$ are the Chebyshev polynomials of 1 st and 2nd order defined by the recurrence $P_{m}(x)=2 x P_{m-1}(x)-P_{m-2}(x) m \geq 0$ provided that $U_{0}(x)=1, U_{1}(x)=x$ and $T_{-2}(x)=-1, T_{-1}(x)=0$, respectively.

Let us now define the standard subdivision of the wheel network. The new vertices are $y_{i}=$ $v_{x_{0} x_{i}}$ and $z_{i}=v_{x_{i} x_{i+1}}$ if $i=1, \ldots, n$. The conductances for the new edges are $2 a=c\left(x_{0}, y_{i}\right)$ and $2 c=c\left(x_{i}, z_{i}\right)$ for $i=1, \ldots, n$. Whereas, the conductance of the remaining edges follows taking into account relation (5).

Observe that $k\left(y_{i}\right)=4 a$ and $k\left(z_{i}\right)=4 c$ for $i=1, \ldots, n$. Moreover, $\alpha(x, y)=\frac{1}{2}$, for every pair of adjacent vertices and $\pi^{S}\left(x_{0}\right)=\frac{n}{2}$ and $\pi^{S}\left(x_{i}\right)=\frac{3}{2}, i=1, \ldots, n$. Then, the expression of the Green kernel for the subdivision network is given next.

Proposition 5.1. Let $\Gamma^{S}$ be the subdivision network of $\Gamma$, and for any $i, j=1, \ldots, n$ consider

$$
g_{i j}(p)=\frac{U_{n-1-|i-j|}(p)+U_{|i-j|-1}(p)}{2 c\left(T_{n}(p)-1\right)} .
$$

Then, the Green kernel for $\Gamma^{S}$ is given by

$$
\begin{aligned}
G^{S}\left(x_{0}, x_{0}\right) & =\frac{n(a+26 c)}{4 a c(3 n+1)^{2}}, & G^{S}\left(x_{0}, x_{i}\right) & =\frac{1}{4 a c(3 n+1)}\left(\frac{n(a+26 c)}{3 n+1}-10 c\right), \\
G^{S}\left(x_{0}, y_{i}\right) & =\frac{1}{4 a c(3 n+1)}\left(\frac{n(a+26 c)}{3 n+1}-6 c\right), & G^{S}\left(x_{0}, z_{i}\right) & =\frac{1}{4 a c(3 n+1)}\left(\frac{n(a+26 c)}{3 n+1}-(a+10 c)\right),
\end{aligned}
$$




$$
\begin{aligned}
G^{S}\left(x_{i}, x_{j}\right) & =g_{i j}(p)+\frac{n(a-34 c)-20 c}{4 a c(3 n+1)^{2}}, \\
G^{S}\left(x_{i}, y_{j}\right) & =\frac{1}{2} g_{i j}(p)+\frac{n(a-34 c)-20 c}{4 a c(3 n+1)^{2}}+\frac{1}{a(3 n+1)}, \\
G^{S}\left(x_{i}, z_{j}\right) & =\frac{1}{2}\left(g_{i j}(p)+g_{i j+1}(p)\right)+\frac{n(a-34 c)-20 c}{4 a c(3 n+1)^{2}}-\frac{1}{4 c(3 n+1)}, \\
G^{S}\left(y_{i}, y_{j}\right) & =\frac{1}{4} g_{i j}(p)+\frac{\varepsilon_{y_{i}}\left(y_{j}\right)}{4 a}+\frac{n(a-34 c)-20 c}{4 a c(3 n+1)^{2}}+\frac{2}{a(3 n+1)}, \\
G^{S}\left(y_{i}, z_{j}\right) & =\frac{1}{4}\left(g_{i j}(p)+g_{i j+1}(p)\right)+\frac{n(a-34 c)-20 c}{4 a c(3 n+1)^{2}}-\frac{a-4 c}{4 a c(3 n+1)}, \\
G^{S}\left(z_{i}, z_{j}\right) & =\frac{p+1}{2} g_{i j}(p)+\frac{n(a-34 c)-20 c}{4 a c(3 n+1)^{2}}-\frac{1}{2 c(3 n+1)}+\frac{\varepsilon_{z_{i}}\left(z_{j}\right)}{4 c} .
\end{aligned}
$$

Proof. The expressions given in the proposition follow from the expression for the Green kernel obtained in Proposition 3.1. We compute one of the cases in order to illustrate the methodology.

Firstly, we compute the constant

$\beta=\frac{1}{(n+m)^{2}} \sum_{s, r \in V} G(s, r) \pi^{S}(r) \pi^{S}(s)+\frac{1}{(n+m)^{2}} \sum_{r \sim s} \frac{1}{k\left(v_{r s}\right)}=\frac{n}{4 a(3 n+1)^{2}}\left[\left(\frac{n-3}{n+1}\right)^{2}+\frac{a}{c}+1\right]$,

where we have taken into account that $\sum_{r \in V} G(s, r)=0$ and hence

$$
\sum_{r \in V} G(s, r) \pi^{S}(r)=\frac{n-3}{2} G\left(s, x_{0}\right) .
$$

Consider $z_{i}=v_{x_{i} x_{i+1}}$ and $z_{j}=v_{x_{j} x_{j+1}}$, then 


$$
\begin{aligned}
G^{S}\left(z_{i}, z_{j}\right) & =\frac{1}{4}\left(G\left(x_{i}, x_{j}\right)+G\left(x_{i+1}, x_{j}\right)+G\left(x_{i}, x_{j+1}\right)+G\left(x_{i+1}, x_{j+1}\right)\right) \\
& -\frac{1}{2(3 n+1)} \sum_{\ell=0}^{n}\left[G\left(x_{j}, x_{\ell}\right)+G\left(x_{j+1}, x_{\ell}\right)+G\left(x_{j}, x_{\ell}\right)+G\left(x_{j+1}, x_{\ell}\right)\right] \pi^{S}\left(x_{\ell}\right) \\
& +\frac{\varepsilon_{z_{j}}\left(z_{i}\right)}{k\left(z_{i}\right)}-\frac{1}{(3 n+1) k\left(z_{i}\right)}-\frac{1}{(3 n+1) k\left(z_{j}\right)}+\beta \\
& =-\frac{n+2}{a(n+1)^{2}}+\frac{2 U_{|i-j|-1}(p)+U_{|i+1-j|-1}(p)+U_{|i-j-1|-1}(p)}{8 c\left(T_{n}(p)-1\right)} \\
& +\frac{2 U_{n-1-|i-j|}(p)+U_{n-1-|i+1-j|}(p)+U_{n-1-|i-j-1|}(p)}{8 c\left(T_{n}(p)-1\right)} \\
& +\frac{n-3}{(3 n+1)(n+1)^{2}}+\frac{\varepsilon_{z_{j}}\left(z_{i}\right)}{4 c}-\frac{2}{(3 n+1) 4 c}+\beta \\
& =\frac{(a+4 c)\left(U_{n-1-|i-j|}(p)+U_{|i-j|-1}(p)\right)}{8 c^{2}\left(T_{n}(p)-1\right)}-\frac{n(5 a+34 c)+2 a+20 c}{4 a c(3 n+1)^{2}}+\frac{\varepsilon_{z_{i}}\left(z_{j}\right)}{4 c} .
\end{aligned}
$$

To end up the section we compute the Kirchhoff index of the standard subdivision graph associated with the wheel $W_{n}$.

Corollary 5.2. The Kirchhoff index of the standard subdivision network of $W_{n}$ is

$$
\mathrm{k}^{S}=\frac{3 n^{2}(a+c)-25 c n}{4 a c}+\frac{n(3 n+1)\left(7 U_{n-1}(p)+2 U_{n-2}(p)+2\right)}{8 c\left(T_{n}(p)-1\right)} .
$$

\section{Acknowledgements}

This work has been partly supported by the Spanish Research Council (Comisión Interministerial de Ciencia y Tecnología,) MTM2014-60450-R.

\section{References}

[1] C. Aráuz. The Kirchhoff indexes of some composite networks. Dis. Appl. Math, 160(1011):1429-1440, 2012.

[2] E. Bendito, A. Carmona, A. M. Encinas, and J. M. Gesto. A formula for the Kirchhoff index. Int. J. Quantum Chem., 108(6):1200-1206, 2008.

[3] E. Bendito, A. Carmona, A. M. Encinas, and J. M. Gesto. Characterization of symmetric M-matrices as resistive inverses. Linear Algebra Appl., 430(4):1336-1349, 2009.

[4] Bendito, E. and Carmona, A. and Encinas, A. M. and Gesto, J. M. and Mitjana, M. Kirchhoff indexes of a network. Linear Algebra Appl., 432(9):2278-2292, 2010. 
[5] E. Bendito and A. Carmona and Encinas A.M. The Kirchhoff indices of join networks. Discrete Appl. Math., 160:24-37, 2012.

[6] C. Bu, B. Yan, X. Zhou, and J. Zhou. Resistance distance in subdivision-vertex join and subdivision-edge join of graphs. Linear Algebra Appl., 458:454-462, 2014.

[7] A. Carmona, A. M. Encinas, and M. Mitjana. Discrete elliptic operators and their Green operators. Linear Algebra Appl., 442:115-134, 2014.

[8] A. Carmona, A. M. Encinas, and M. Mitjana. Discrete elliptic operators and their Green operators. Linear Algebra Appl., 442:115-134, 2014.

[9] H. Chen. Random walks and the effective resistance sum rules. Discrete Appl. Math., 158(15):1691-1700, 2010.

[10] H. Chen and F. Zhang. Resistance distance and the normalized Laplacian spectrum. Discrete Appl. Math., 155(5):654-661, 2007.

[11] W. Ellens, F.M. Spieksma, P. Van Mieghem, A. Jamakovic, R.E. Kooij. Effective graph resistance. Linear Algebra Appl., 435:2491-2506, 2011.

[12] X. Gao, Y. Luo, and W. Liu. Kirchhoff index in line, subdivision and total graphs of a regular graph. Discrete Appl. Math., 160(4-5):560-565, 2012.

[13] A. Ghosh, S. Boyd, and A. Saberi. Minimizing effective resistance of a graph. SIAM Review, $50(1): 37-66,2008$.

[14] S. Huang, J. Zhou and C. Bu. Some results on Kirchhoff index and degree-Kirchhoff index. MATCH Commun. Math. Comput. Chem., 75:207-222, 2016.

[15] D.J. Klein and M. Randić. Resistance distance. J. Math. Chem., 12(1):81-95, 1993.

[16] L. Sun, W. Wang, J. Zhou, and C. Bu. Some results on resistance distances and resistance matrices. Linear Multilinear Algebra, 63(3):523-533, 2015.

[17] W. Xiao and I. Gutman. Resistance distance and Laplacian spectrum. Theor. Chem. Acc., 110(4):284-289, 2003.

[18] Y. Yang. The Kirchhoff index of subdivisions of graphs. Discrete Appl. Math., 171(0):153 $157,2014$.

[19] Y. Yang, D.J. Klein. A recursion formula for resistance distance and its applications. Discrete Appl. Math., 161:2702-2715, 2013. 